\title{
Viscoelastic-plastic creep constitutive model of frozen soil
}

Xueye Cao PhD*

School of Civil Engineering, Chang'an University, Xi'an, China

Junhai Zhao PhD

Professor, School of Civil Engineering, Chang'an University, Xi'an, China
Yan Li PhD

Lecturer, School of Geology Engineering and Geomatics, Chang'an University, Xi'an, China

In accordance with the twin-shear unified strength theory and the improved Nishihara model, a viscoelastic-plastic creep constitutive function of frozen soil has been established based on the associated flow rules, and the viscoelastic-plastic soft matrix is further derived from numerical calculations. The results show that the viscoelastic-plastic creep constitutive function has considerable influence on the non-linearity and the intermediate principal stress of materials, and it can describe the creep properties of frozen soil as a unified solution. The proposed model herein is applicable to the creep study of frozen soil.

\section{Notation}

$b$ parameter that reflects the damage extent of the intermediate principal shear stress and the corresponding normal stress

$E_{1}, E_{\mathrm{H}}$ elasticity moduli (MPa)

$F \quad$ yield function of the twin-shear unified strength theory

$G_{1} \quad$ viscous elastic shear modulus (MPa)

$G_{\mathrm{H}} \quad$ instantaneous elastic shear modulus (MPa)

$H_{1}, H_{2}$ viscoelastic coefficients

$I_{1} \quad$ first invariant of stress tensor (hydrostatic stress)

$\mathrm{J}_{2} \quad$ second invariant of deviator stress

$\alpha \quad$ ratio of tension strength and compression strength, $\alpha=$ $\sigma_{\mathrm{s}} / \sigma_{\mathrm{c}}$

$\varepsilon \quad$ total strain

$\varepsilon_{\mathrm{t}}^{\mathrm{e}} \quad$ elastic strain component

$\varepsilon_{\mathrm{t}}^{\mathrm{p}} \quad$ plastic strain component

$\eta_{1}, \eta_{2}$ viscosity coefficients

$\theta \quad$ corresponding angle of the twin-shear stress parameter $\mu_{\tau}=\tau_{12} / \tau_{13}$ or $\mu_{\tau}^{\prime}=\tau_{23} / \tau_{13}$

$\sigma_{\mathrm{c}} \quad$ compressive yield limitation

$\sigma_{\mathrm{s}} \quad$ tensile yield limitation

\section{Introduction}

Frozen soil is a type of special geotechnical material; the mechanical characteristic of frozen soil is sensitive to temperature, water content and pressure. ${ }^{1,2}$ As its interior contains unfrozen soil and ice, the internal stress and strain are the result of accumulation over time, which means that deformation grows with time under constant load or stress evolves with time under constant deformation. $^{3}$ With the development of freeze sinking and engineering construction in cold regions, more and more engineering problems are exposed. For example, many freezing pipes rupture because of large creep deformation, and numerous foundations of high-speed roads, railways and large buildings crack due to the creep deformation of frozen soil, which severely affects the normal use of buildings and structures. Therefore, research on creep properties of frozen soil, especially creep constitutive model, has important theoretical significance and engineering value. ${ }^{4,5}$

Research on creep constitutive model of frozen soil has been conducted since the $1960 \mathrm{~s}$. Li et al. ${ }^{6}$ established a statistical constitutive model by using the continuum damage theory and introducing the Drucker-Prager criterion as its parameter of macroscopic element strength. Yuan ${ }^{7}$ constructed a viscoelastic-plastic constitutive model on the basis of the Nishihara model, which adds a viscoelastic element and parabolic yield function instead of plastic yield. Wang et $a l^{8}$ found that the Nishihara model can be improved by substituting the non-linear Newtonian element for the linear one and derived a soft matrix of viscoelastic-plastic materials on the basis of von Mises yield criterion. $\mathrm{Li}$ and Wang $^{9}$ deduced a viscoelastic-plastic damagecoupling constitutive function based on thermodynamics, statistical damage theory and the associated flow rules on the assumption that a new form of frozen soil microunit strength follows the improved Mohr-Coulomb criterion. Although previous research has provided solid theoretical foundations for creep characteristic studies of frozen soil, some deficiencies still exist. Few studies considered the influence of the intermediate principal stress on the creep deformation of frozen soil, and the proposed constitutive model in the present study can be used for one or several special materials.

Based on the results that have been obtained in earlier studies, the non-linear Newtonian element can be substituted for the linear one to improve the Nishihara model. Further, based on the twinshear unified strength theory and the associated flow rules, the viscoelastic-plastic creep constitutive function has been deduced and a viscoelastic-plastic soft matrix is derived, which is applicable to the subsequent numerical calculations. 


\section{The creep constitutive function of frozen soil}

\subsection{The twin-shear unified strength theory}

Based on the twin-shear unit and the twin-shear yield criterion, $\mathrm{Yu}^{10}$ established the twin-shear unified strength theory in 1991 by considering all the stresses acting on the twin-shear unit and their effects on damage in different materials. The twin-shear unified strength theory is suitable for different materials and has different mathematical expressions. The stress invariant expression is ${ }^{10}$

$$
\begin{array}{rlrl}
F & =\frac{1-\alpha}{3 \alpha} I_{1}+\frac{1-b}{1+b} J_{2}^{1 / 2} \sin \theta+\frac{2+\alpha}{\alpha 3^{1 / 2}} J_{2}^{1 / 2} \cos \theta=\sigma_{\mathrm{c}} & 0 \leq \theta \leq \theta_{\mathrm{b}} \\
\text { 1. } & F^{\prime}=\frac{1-\alpha}{3 \alpha} I_{1}+\frac{\alpha+\alpha b+b}{\alpha(1+b)} J_{2}^{1 / 2} \sin \theta+\frac{2+\alpha+\alpha b-b}{\alpha 3^{1 / 2}(1+b)} J_{2}^{1 / 2} \cos \theta=\sigma_{\mathrm{c}} & 0 \leq \theta \leq \theta_{\mathrm{b}}
\end{array}
$$

where $\alpha$ is the ratio of tension strength and compression strength, $\alpha=\sigma_{\mathrm{s}} / \sigma_{\mathrm{c}} ; I_{1}$ is the first invariant of stress tensor (hydrostatic stress); $J_{2}$ is the second invariant of deviator stress; $b$ is the parameter that reflects the damage extent of the intermediate principal shear stress and the corresponding normal stress; $\sigma_{\mathrm{s}}$ and $\sigma_{\mathrm{c}}$ are the tensile yield limitation and compressive yield limitation, respectively; $\theta$ is the corresponding angle of the twin-shear stress parameter $\mu_{\tau}=\tau_{12} / \tau_{13}$ or $\mu_{\tau}^{\prime}=\tau_{23} / \tau_{13}$; and $\theta_{\mathrm{b}}$ can be obtained by the equation of $F=F^{\prime}$.

2. $\theta_{\mathrm{b}}=\arctan \frac{3^{1 / 2}}{2 \alpha+1}$

Equation 1 consists of a series of failure criteria that vary with $b$; it can be simplified to many prevailing failure criteria. For example, it reduces to Mohr-Coulomb failure criterion with $b=$ 0 . The twin-shear yield criterion is obtained with $b=1$, and a series of new convex non-linear strength criterion are reduced with $0<b<1$. Hence, the twin-shear unified strength criterion should not be regarded as a single special failure criterion but a theoretical system that can be applied to a wide variety of materials.

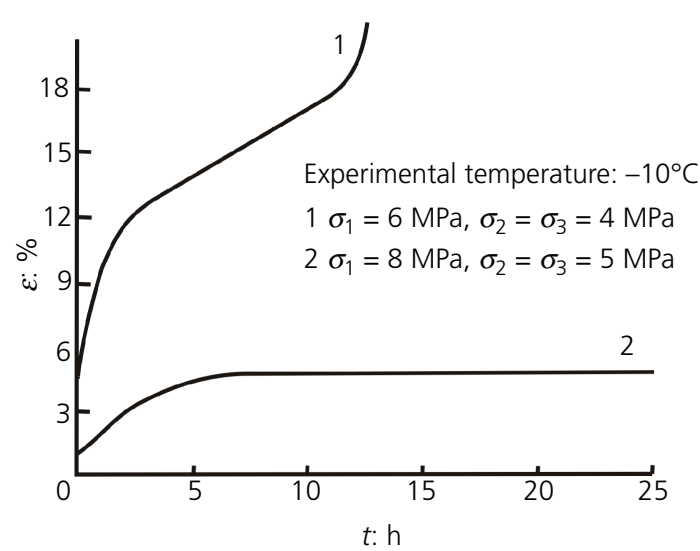

Figure 1. Typical creep curves of frozen soil

\subsection{The creep characteristics of frozen soils}

According to previous experimental results, the typical creep deformation curve is shown in Figure $1 .^{11}$

Figure 1 shows that the laws of creep deformation of frozen soils are as follows: $(a)$ instantaneous strain occurs at the initiation of loading, which is $5-10 \%$ of total creep deformation; $(b)$ when the deviatoric stress is low, there only appear unsteady creep and steady creep, namely, the first stage and the second stage; and (c) when the deviatoric stress is high, an accelerated creep appears, namely, the third stage.

\subsection{The viscoelastic-plastic creep constitutive function of frozen soil}

To describe the non-linear viscoelastic plasticity of frozen soil, the Nishihara model is improved by substituting the non-linear Newtonian element for the linear one. The researchers assume that the yield criterion of materials is governed by the unified strength theory, as shown in Figure 2. The improved Nishihara model can be used to analyze the non-linear viscoelastic-plastic creep of frozen soil.

The single-axis viscoelastic-plastic creep constitutive equation is

3. $\varepsilon=\frac{1}{E_{\mathrm{H}}}+\frac{\sigma}{E_{1}}\left(1-e^{\left(E_{1} / \eta_{1}\right) t}\right)+\langle F\rangle \frac{t^{2}}{\eta_{2}}$

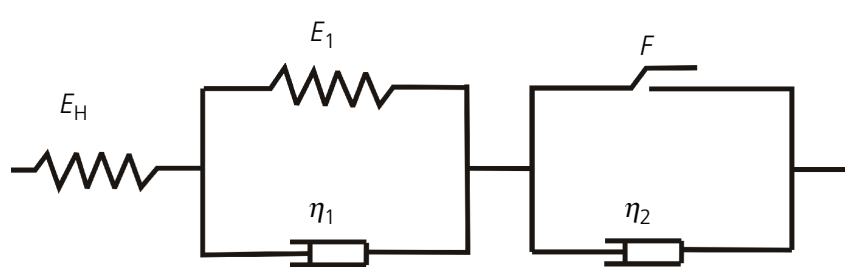

Figure 2. The viscous elastic-plastic creep constitutive model for frozen soil 
where $F$ is the yield function of the twin-shear unified strength theory; $E_{1}$ and $E_{\mathrm{H}}$ are the elasticity moduli (MPa); $\eta_{1}$ and $\eta_{2}$ are the viscosity coefficients, the units of which are, respectively, megapascal-minutes and megapascal-square minutes.

The creep constitutive model, in three-dimensional (3D) stress state, is difficult to describe with visual physical components. Research ${ }^{3}$ shows that the creep constitutive equation is in accord with the onedimensional model. By using the method of analogy, the constitutive model of frozen soil, in 3D stress state, is obtained as

4.

$$
\begin{aligned}
\varepsilon_{i j}= & {\left[\frac{1}{2 G_{\mathrm{H}}}+\frac{1}{2 G_{1}}\left(1-e^{\left(G_{1} / H_{1}\right) t}\right)\right]\left\{\sigma_{i j}\right\} } \\
& +\left\langle\frac{F}{F_{0}}\right\rangle\left(\frac{t^{2}}{2 H_{2}}\right) \frac{\partial Q}{\partial\left\{\sigma_{i j}\right\}}
\end{aligned}
$$

where $G_{\mathrm{H}}$ is the instantaneous elastic shear modulus (MPa); $G_{1}$ is the viscous elastic shear modulus (MPa); and $H_{1}$ and $H_{2}$ are the viscoelastic coefficients, the units of which are, respectively, megapascal-minutes and megapascal-square minutes.

\subsection{The viscoelastic-plastic creep soft matrices}

During the process of calculating the non-linear continuum deformation, the total strain $\varepsilon$ is usually divided into the elastic strain component $\varepsilon_{\mathrm{t}}^{\mathrm{e}}$ (including instantaneous elastic strain and linear and non-linear viscous elastic strains) and the plastic strain component $\varepsilon_{\mathrm{t}}^{\mathrm{p}}$ (including instantaneous plastic strain and viscous plastic strain). Thus, the expression of the total strain is ${ }^{12}$

\section{5. $\varepsilon=\varepsilon_{\mathrm{t}}^{\mathrm{e}}+\varepsilon_{\mathrm{t}}^{\mathrm{p}}$}

\subsubsection{The viscoelastic soft matrix}

The elastic strain of frozen soil is obtained by Equation 4 as

6. $\varepsilon_{\mathrm{t}}^{\mathrm{e}}=\left[\frac{1}{2 G_{\mathrm{H}}}+\frac{1}{2 G_{1}}\left(1-e^{\left(G_{1} / H_{1}\right) t}\right)\right]\left\{\sigma_{i j}\right\}$

$G_{\mathrm{t}}$ is defined as

$$
G_{\mathrm{t}}=\frac{1}{\frac{1}{2 G_{\mathrm{H}}}+\frac{1}{2 G_{1}}\left(1-e^{\left(G_{1} / H_{1}\right) t}\right)}
$$

Then, the following is obtained

7. $\varepsilon_{\mathrm{t}}^{\mathrm{e}}=\frac{1}{G_{\mathrm{t}}}\{\sigma\}=\left[\mathbf{C}^{\mathrm{e}}\right]\{\sigma\}$ where $\left[\mathbf{C}^{\mathrm{e}}\right]$ is the elastic soft matrix

$\left[\mathbf{C}^{\mathrm{e}}\right]=\frac{1}{G_{\mathrm{t}}}\left[\begin{array}{cccccc}1 & -\mu & -\mu & 0 & 0 & 0 \\ -\mu & 1 & -\mu & 0 & 0 & 0 \\ -\mu & -\mu & 1 & 0 & 0 & 0 \\ 0 & 0 & 0 & 2(1+\mu) & 0 & 0 \\ 0 & 0 & 0 & 0 & 2(1+\mu) & 0 \\ 0 & 0 & 0 & 0 & 0 & 2(1+\mu)\end{array}\right]$

8.

\subsubsection{The viscoplastic soft matrix}

Similarly, the plastic strain of frozen soil can also be calculated by Equation 4 as

9.

$$
\varepsilon_{\mathrm{t}}^{\mathrm{p}}=\left\langle\frac{F}{F_{0}}\right\rangle\left(\frac{t^{2}}{2 H_{2}}\right) \frac{\partial Q}{\partial\left\{\sigma_{i j}\right\}}
$$

where $F$ is the yield function, which is determined by Equation 1; for geotechnical materials, $F_{0}=1$.

The plastic matrix can be derived by the following formula

10. $\frac{\partial F}{\partial\{\sigma\}}=\frac{\partial F}{\partial I_{1}} \frac{\partial I_{1}}{\partial\{\sigma\}}+\frac{\partial F}{\partial J_{2}^{1 / 2}} \frac{\partial J_{2}^{1 / 2}}{\partial\{\sigma\}}+\frac{\partial F}{\partial \theta} \frac{\partial \theta}{\partial\{\sigma\}}$

where

11. $\frac{\partial I_{1}}{\partial\{\sigma\}}=[\mathbf{R}]=\left[\begin{array}{llllll}1 & 1 & 1 & 0 & 0 & 0\end{array}\right]^{T}$

$$
\frac{\partial J_{2}}{\partial\{\sigma\}}=[\mathbf{S}]=\frac{1}{2 J_{2}^{1 / 2}}\left[\begin{array}{llllll}
S_{x} & S_{y} & S_{z} & 2 \tau_{y z} & 2 \tau_{z x} & 2 \tau_{x y}
\end{array}\right]^{T}
$$

$$
\begin{aligned}
\frac{\partial J_{3}}{\partial\{\sigma\}}= & {[\mathbf{Q}] } \\
= & {\left[\left(S_{y} S_{z}-\tau_{y z}^{2}+\frac{J_{2}}{3}\right)\left(S_{x} S_{z}-\tau_{z x}^{2}+\frac{J_{2}}{3}\right)\right.} \\
& \left(S_{x} S_{y}-\tau_{x y}^{2}+\frac{J_{2}}{3}\right) 2\left(\tau_{z x} \tau_{x y}-\tau_{y z} S_{x}\right) \\
& \left.2\left(\tau_{x y} \tau_{y z}-\tau_{z x} S_{y}\right) 2\left(\tau_{y z} \tau_{z x}-\tau_{x y} S_{z}\right)\right]^{T}
\end{aligned}
$$


$C_{1}, C_{2}$ and $C_{3}$ are defined as

$$
\begin{aligned}
& C_{1}=\frac{\partial F}{\partial I_{1}} \\
& C_{2}=\frac{\partial F}{\partial J_{2}^{1 / 2}}-\frac{\tan 3 \theta}{J_{2}^{1 / 2}} \frac{\partial F}{\partial \theta} \\
& C_{3}=-\frac{3^{1 / 2}}{2 \cos 3 \theta\left(J_{2}^{3}\right)^{1 / 2}} \frac{\partial F}{\partial \theta}
\end{aligned}
$$

Then, the following is obtained.

15. $\frac{\partial F}{\partial\{\sigma\}}=C_{1}[\mathbf{R}]+C_{2}[\mathbf{S}]+C_{3}[\mathbf{Q}]$

When $0 \leq \theta \leq \theta_{\mathrm{b}}$,

$$
\begin{aligned}
C_{1}= & \frac{1-\alpha}{3 \alpha} \\
C_{2}= & \frac{1-b}{1+b} \sin \theta+\frac{2+\alpha}{\alpha 3^{1 / 2}} \cos \theta \\
& -\tan 3 \theta\left[\frac{1-b}{1+b} \cos \theta-\frac{2+\alpha}{\alpha 3^{1 / 2}} \sin \theta\right] \\
\text { 16. } C_{3}= & -\frac{3^{1 / 2}}{2 J_{2} \cos 3 \theta}\left[\frac{1-b}{1+b} \cos \theta-\frac{2+\alpha}{\alpha 3^{1 / 2}} \sin \theta\right]
\end{aligned}
$$

When $\theta_{\mathrm{b}} \leq \theta \leq 60^{\circ}$,

$$
\begin{aligned}
C_{1}^{\prime}= & \frac{1-\alpha}{3 \alpha} \\
C_{2}^{\prime}= & \frac{\alpha+\alpha b+b}{\alpha(1+b)} \sin \theta+\frac{2+\alpha+\alpha b-b}{\alpha 3^{1 / 2}(1+b)} \cos \theta \\
& -\tan 3 \theta\left[\frac{\alpha+\alpha b+b}{\alpha(1+b)} \cos \theta-\frac{2+\alpha+\alpha b-b}{\alpha 3^{1 / 2}(1+b)} \sin \theta\right] \\
C_{3}^{\prime}= & -\frac{3^{1 / 2}}{2 J_{2} \cos 3 \theta}\left[\frac{\alpha+\alpha b+b}{\alpha(1+b)} \cos \theta\right. \\
& \left.-\frac{2+\alpha+\alpha b-b}{\alpha 3^{1 / 2}(1+b)} \sin \theta\right]
\end{aligned}
$$

17.

The viscoplastic creep strain increment can be calculated by the following formula

18. $\Delta \varepsilon^{\mathrm{p}}=\dot{\varepsilon}^{\mathrm{p}} \Delta t$
Based on Equation 9, the following is obtained

19. $\dot{\varepsilon}^{\mathrm{p}}=\frac{F t}{H_{2}} \frac{\partial F}{\partial\{\sigma\}}$

By substituting Equation 19 into Equation 18, the viscoplastic strain increment along with time step is obtained as follows

20. $\Delta \varepsilon^{\mathrm{p}}=\frac{F t}{H_{2}} \Delta t \frac{\partial F}{\partial\{\sigma\}}$

Furthermore, the viscoplastic soft matrix is derived by the aforementioned equation

21.

$$
\left[\mathbf{C}^{\mathrm{p}}\right]=\frac{F t}{H_{2}}\left(C_{1}[\mathbf{R}]+C_{2}[\mathbf{S}]+C_{3}[\mathbf{Q}]\right)
$$

\subsection{The unified solution of viscous elastic-plastic soft matrices}

By combining Equations 8 and 21, the viscoelastic-plastic soft matrices based on the twin-shear unified strength theory can be obtained, from which many special viscoelastic-plastic soft matrices can be degenerated. For example, when $C_{1}=0, C_{2}=3^{1 / 2}$ and $C_{3}=0$, the twin-shear unified strength theory degenerates to a von Mises yield criterion, and the von Mises-based viscoplastic soft matrix is obtained by Equations 11-13 and 21, which is the same as in the study by Wang et al. ${ }^{8}$

According to a unified mechanical model, the twin-shear unified strength theory considers all the stress components and their effects on damage in different materials. It includes a series of yield criteria and adapts to the elastic-plastic analysis of various materials. Therefore, the viscoelastic-plastic constitutive function of frozen soil and the viscoelastic-plastic soft matrices, suitable for numerical calculations herein, can be used as the unified solution to analyze the creep characteristics of frozen soil.

\section{Conclusions}

(a) According to the twin-shear unified strength theory and the improved Nishihara model, the viscoelastic-plastic creep constitutive function was established and the viscoelastic-plastic soft matrices were derived. The proposed model has considerable influence on non-linearity and intermediate principal stress, and the soft matrices can be added into a large non-linear software to analyze the creep characteristics of frozen soil.

(b) Results in this paper can be extended into various components that are suitable for various materials; they can be considered the unified solution of viscoelastic-plastic creep constitutive model of frozen soil. 


\section{Acknowledgements}

The authors would like to acknowledge the financial support from the National Natural Science Foundation of China (number 41202191) and The Cultivation Project of Excellent Doctorial Dissertations of Chang'an University (number 310828150018).

\section{REFERENCES}

1. Yang YG, Gao F, Cheng HM and Hou P (2016) Energy dissipation and failure criterion of artificial frozen soil. Cold Regions Science and Technology 129: 137-144.

2. Bronfenbrener $L$ and Bronfenbrener $R$ (2012) A temperature behavior of frozen soils: field experiments and numerical solution. Cold Regions Science and Technology 79-80: 84-91.

3. Li DW, Wang RH and Lin B (2007) Viscoelastic plastic model of frozen soil and numerical calculation soft matrix applied. Journal of Glaciology and Geocryology 29(2): 322-326.

4. Zhang XD, Zhang SG, Li YJ and Huo BR (2004) Testing study on triaxial creep properties of frozen soil to determine thickness of frozen wall. Chinese Journal of Rock Mechanics and Engineering 23(3): 395-400.

5. Wang SH, Qi JL, Yin ZY, Zhang JM and Ma W (2014) A simple rheological element based on creep model for frozen soils. Cold Regions Science and Technology 106-107: 47-54.
6. Li QZ, Lai YM, Xu XT, Yang YG and Chang XX (2010) Triaxial strength distribution of warm frozen soil and its damage statistical constitutive model. Journal of Glaciology and Geocryology 32(6): 1235-1241.

7. Yuan WH (2013) Back analysis of viscoelasto-plastic constitutive parameters of artificial frozen soil. Rock and Soil Mechanics 34(11) 3091-3095.

8. Wang RH, Li DW and Wang XX (2006) Improved Nishihara model and realization in ADINA FEM. Rock and Soil Mechanics 27(11): 1954-1958.

9. Li DW and Wang RH (2007) Frozen soil creep model based on statistical damage theory. Chinese Journal of Applied Mechanics 24(4): 133-136.

10. Yu MH (2004) Unified Strength Theory and Its Applications. Springer, Berlin, Germany.

11. Li DW, Wang RH and Hu P (2007) Study of frozen soil damagecoupling constitutive function. Journal of Glaciology and Geocryology 29(3): 446-449.

12. Wang CW and Li DW (2013) Study and verification of laboratory prepared frozen soil viscoelastic-plastic damage coupling constitutive model. Journal of Glaciology and Geocryology 35(5): 1219-1223.

\section{How can you contribute?}

To discuss this paper, please submit up to 500 words to the journal office at journal@ice.org.uk. Your contribution will be forwarded to the author(s) for a reply and, if considered appropriate by the editor-in-chief, it will be published as a discussion in a future issue of the journal.

ICE Science journals rely entirely on contributions from the field of materials science and engineering. Information about how to submit your paper online is available at www.icevirtuallibrary.com/page/authors, where you will also find detailed author guidelines. 